\title{
A NOTE ON SOME ELEMENTARY MEASURES OF ALGEBRAIC INDEPENDENCE
}

\author{
ROBERT TUBBS
}

(Communicated by William Adams)

\begin{abstract}
We investigate the algebraic independence of some numbers associated with elliptic functions when one of the numbers is a "Liouville-type" number. Suppose $\wp(z)$ is a Weierstrass elliptic function with algebraic invariants and $\beta$ is an algebraic number, not belonging to the field of multiplications for $\wp(z)$. We establish the algebraic independence of $\wp(u)$ and $\wp(\beta u)$ (respectively, of $u$ and $\wp(\beta u)$ ) when $\wp(u)$ (respectively, $u$ ) is a "Liouville-type" number. We also give quantitative versions of these results.
\end{abstract}

In this paper we study the algebraic independence of two numbers associated with Liouville-type numbers and points on an elliptic curve. The motivation for this study comes from two sources: one was to provide an elliptic analogue to a result of N. I. Feldman [3] concerning the algebraic independence of $\alpha$ and $\alpha^{\beta}$ when $\alpha$ is "well-approximated" in a certain sense and $\beta$ is algebraic, irrational; the other was to continue the investigation of the present author in [8] which provided a measure for the transcendence of a special value of a Weierstrass elliptic function.

Some generalizations and extensions of Feldman's work have already been given. Specifically W. D. Brownawell [1] and Brownawell and M. Waldschmidt [2] have examined the algebraic independence of $\alpha, \alpha^{\beta}, \alpha^{\beta^{2}}$ when $\alpha$ is "well approximated" and $\beta$ is cubic, and the present author has provided an elliptic analogue to Brownawell's result [7]. Additionally M. Laurent [5] has improved the $\alpha$ and $\alpha^{\beta}$ result of Feldman, and it is along these lines that we work in the elliptic case.

Let $\wp(z)$ denote a Weierstrass elliptic function with its invariants $g_{2}$ and $g_{3}$ being algebraic. (Recall that $\wp(z)$ satisfies the differential equation: $\left(\wp^{\prime}(z)\right)^{2}=$ $\left.4 \wp^{3}(z)-g_{2} \wp(z)-g_{3}\right)$. The function $\wp(z)$ is doubly periodic and meromorphic. Let $\mathscr{O} \subseteq \mathbf{C}$ denote the ring of endomorphisms of the elliptic curve associated with $\wp(z)$ and let $K$ denote the field of fractions of $\mathscr{O}$.

For an algebraic number $\alpha$ we let $h t(\alpha)$ denote the maximum absolute value of the coefficients of the minimal polynomial of $\alpha$ over $Z$ and let $\operatorname{deg} \alpha$ denote

Received by the editors October 17, 1988 and, in revised form, June 19, 1989.

1980 Mathematics Subject Classification (1985 Revision). Primary 11J89; Secondary 11 K60. 
the degree of that polynomial. With these conventions we can now make the notation of "well approximated", referred to above, precise.

Suppose that $f$ is a function defined on the set of nonnegative integers. For the purpose of this paper we say that a complex number $\zeta$ is $f$-approximable if for some positive integer $d_{0}$ and an infinite number of positive integers $T$ there exists an algebraic number $a_{T}$ satisfying

$$
\operatorname{deg} a_{T} \leq d_{0}, \quad h t\left(a_{T}\right) \leq e^{T}
$$

and

$$
0<\left|a_{T}-\zeta\right|<\exp (-f(T)) .
$$

We let $\left\{a_{T_{j}}\right\}_{j=1}^{\infty}$ denote the sequence of algebraic numbers associated with the approximation of $\zeta$.

In each theorem below assume that $\wp(z)$ is a Weierstrass elliptic function with algebraic invariants and that $\beta$ is a nonzero algebraic number.

Theorem 1. Suppose $\beta \notin K$. If for some $\varepsilon>2, \wp(u)$ is $T^{\varepsilon}$-approximable, then $\wp(u)$ and $\wp(\beta u)$ are algebraically independent.

Moreover, we have a quantitative version of Theorem 1 which depends more subtlely on the sequence $\left\{a_{T}\right\}$ approximating $\wp(u)$.

Theorem 2. Suppose $\beta \notin K$ and that for some $\varepsilon>2, \wp(u)$ is $T^{\varepsilon}$-approximable. Suppose that $P(x, y)$ is a nonzero integral polynomial of total degree at most $d_{p}$ and height at most $h_{p}$. Put

$M=\left(\frac{1}{\varepsilon-1}\right)^{1 / \varepsilon} d_{0}^{14 /(\varepsilon-2)} d_{p}^{8 /(\varepsilon-2)}\left(\log h_{p}\right)^{1 /(\varepsilon-1)}\left(\log d_{0}+\log d_{p}+\log \log h_{p}\right)^{3 /(\varepsilon-2)}$.

Then there exists a constant $S_{1}$, depending only on $d_{0}, \beta, \wp$, and $\varepsilon$, such that if $\min \left\{d_{p}, h_{p}\right\} \geq S_{1}$ one has

$$
|P(\wp(u), \wp(\beta u))|>\exp \left(-t^{\varepsilon} M^{\varepsilon}\right),
$$

where

$$
t=\frac{T_{k+1}}{T_{k}}, \quad k=\max \left\{j: T_{j} \leq M\right\} .
$$

We also consider the values $u$ and $\wp(\beta u)$ where $u$ is "well approximated" by algebraic numbers. This is the object of the next two theorems.

Theorem 3. Suppose $\beta \neq 0$. If for some $\varepsilon>3, u$ is $T^{\varepsilon}$-approximable, then $u$ and $\wp(\beta u)$ are algebraically independent.

As did Theorem 1, Theorem 3 also has a quantitative version.

Theorem 4. Suppose $\beta \neq 0$ and that for some $\varepsilon>3, u$ is $T^{\varepsilon}$-approximable. Suppose that $P(x, y)$ is a nonzero integral polynomial of total degree at most $d_{p}$ and height at most $h_{p}$. Put

$$
M_{1}=\left(\frac{1}{\varepsilon-2}\right)^{1 / \varepsilon} d_{0}^{10 /(\varepsilon-3)} d_{p}^{6 /(\varepsilon-3)}\left(\log h_{p}\right)^{1 /(\varepsilon-2)} .
$$


Then there exists a constant $S_{2}$, depending only on $d_{0}, \beta, \wp$, and $\varepsilon$, such that if $\min \left\{d_{p}, h_{p}\right\} \geq S_{2}$, one has

$$
|P(u, \wp(\beta u))|>\exp \left(-t_{1}^{\varepsilon} M_{1}^{\varepsilon}\right),
$$

where

$$
t_{1}=\frac{T_{k+1}}{T_{k}}, \quad k=\max \left\{j: T_{j} \leq M_{1}\right\} .
$$

The proofs of these theorems are based on three lemmas. The first of these is very elementary, the second is still rather basic, but the third is a very deep result due to Patrice Philippon and Michel Waldschmidt.

Lemma 1. There exist constants $k_{1}$ and $k_{2}$ depending on $u$ and $\wp(z)$ such that if $|a-\wp(u)|<k_{1}$, then there exists $w \in \mathbf{C}$ with $\wp(w)=a$ and $|w-u|<$ $k_{2}|\wp(w)-\wp(u)|$, provided $\wp^{\prime}(u) \neq 0$.

Proof. Elementary, using the existence of a locally (at $u$ ) analytic inverse of $\wp(z)$.

As hinted at above the next lemma is not quite as elementary.

Lemma 2. Suppose $P(X) \in \mathbf{Z}[X]$ is a nonzero polynomial with $d=\operatorname{deg} p$ and $h=h t P$. Let $\zeta_{i}(1 \leq i \leq k)$ denote the distinct roots of $P$ with multiplicities $m_{i}$. Then for any $\theta \in \mathbf{C}$

$$
\min _{1 \leq i \leq k}\left|\theta-\zeta_{1}\right|^{m_{i}} \leq(2 d) \cdot 2^{d} \cdot(1+d)^{2 d} e^{2 d^{2}} h^{2 d}|P(\theta)| .
$$

Proof. See Lemma 5.3.7 of [9].

To state our third lemma we need a bit of notation. Suppose that $u_{1}, \ldots, u_{n}$ are complex numbers such that each of $\wp\left(u_{1}\right), \ldots, \wp\left(u_{n}\right)$ is algebraic, and suppose further that $\beta_{0}, \beta_{1}, \ldots, \beta_{n}$ are arbitrary algebraic numbers. Put $L=$ $\mathbf{Q}\left(g_{2}, g_{3}, \beta_{0}, \ldots, \beta_{n}, \wp\left(u_{1}\right), \ldots, \wp\left(u_{n}\right)\right)$ and $D=[L: \mathbf{Q}]$. Moreover let $B, E, V_{1}, \ldots, V_{n}$ be real numbers satisfying

$$
\begin{gathered}
\log V_{i} \geq \min \left\{\log h t\left(\wp\left(u_{i}\right)\right),\left|u_{i}\right|^{2} / D\right\}, \quad 1 \leq i \leq n, \\
B \geq \max \left\{D \log V_{1}, \ldots, D \log V_{n}, h t\left(\beta_{0}\right), \ldots, h t\left(\beta_{n}\right)\right\} \\
E \leq \min \left\{B^{D}, \min _{1 \leq i \leq n}\left\{e\left(D \log V_{i}\right)^{1 / 2} /\left|u_{i}\right|\right\}\right\} .
\end{gathered}
$$

Lemma 3. Let $\Lambda=\beta_{0}+\beta_{1} u_{1}+\cdots+\beta_{n} u_{n}$. There exists a constant $C_{3}>0$ depending only on $n, g_{2}$, and $g_{3}$ such that if $\Lambda \neq 0$, we have

$$
|\Lambda|>\exp \left(-C_{3} D^{2 n+2}(\log B)^{n+1}\left(\prod_{i=1}^{n} \log V_{i}\right) \log (D E)(\log E)^{-2 n-1}\right) .
$$

Proof. This is Theorème 1.1 of [6] with $d_{1}=0$ and $h(x)=\log h t(x)$.

We present here a complete proof of Theorem 2 and then only indicate changes which are necessary to establish Theorem 4. Each of these proofs is a 
variant of the proof of Theorème 1 of [5]. Clearly Theorem 1 and Theorem 3 follow from these results. The lower case constants $c_{1}, \ldots, c_{13}$ which appear below are effective and depend at most on $u, \beta$, and $\wp(z)$.

Proof of Theorem 2. We begin by noting that $\wp^{\prime}(\beta u) \neq 0$; or, equivalently, $E(\wp(\beta u)) \neq 0$ where $E(X)=4 X^{3}-g_{2} X-g_{3}$. To obtain this let $R_{T}(X)$ denote the minimal polynomial of $a_{T}$ over $\mathbf{Q}$ where degree $R_{T} \leq d_{0}$ and height $R_{T} \leq e^{T}$. Since $R_{T}\left(a_{T}\right)=0$ we obtain

$$
\left|R_{T}(\wp(u))\right|=\left|R_{T}(\wp(u))-R_{T}\left(a_{T}\right)\right| \leq e^{T}\left(1+d_{0}\right)^{2}(1+|\wp(u)|)^{d_{0}} e^{-T^{\varepsilon}} .
$$

If $E(\wp(\beta u))=0$, then $\wp(\beta u) \in \overline{\mathbf{Q}}$ and from $\beta \notin K$ with $\wp(u)=\wp\left(\beta^{-1} \beta u\right)$ we deduce from the Theorem of [8] that

$$
\left|R_{T}(\wp(u))\right| \geq e^{-c_{1} d_{0}^{6}\left(d_{0}+T\right)^{2}\left(\log \left(d_{0}+T\right)\right)^{14}} .
$$

Since $\varepsilon>2$, these bounds are incompatible as $T$ approaches infinity.

Suppose that $P(x, y) \in \mathbf{Z}[x, y]$ is a nonzero polynomial of total degree at most $d_{p}$ and height at most $h_{p}$ with

$$
|P(\wp(u), \wp(\beta u))| \leq \exp \left(-t^{\varepsilon} M^{\varepsilon}\right),
$$

with $t$ and $M$ as in the statement of the theorem.

Take $S_{1}$ sufficiently large so that $M \geq T_{1}$. Then by our choice of $k=$ $\max \left\{j: T_{j} \leq M\right\}$ we have

$$
T_{k} \leq M \leq T_{k+1} \leq t M .
$$

Put $T=T_{k+1}$ and let $a_{T}=\alpha_{1}, \ldots, \alpha_{\delta}$ denote the conjugates of $a_{T}$ (here $\delta$ denotes the degree of $a_{T}$ so that $\delta \leq d_{0}$ ). Also, let $q$ denote a denominator for $a_{T}$, so that $|q|<e^{T}$.

Then

$$
Q(y)=q^{\delta d_{p}} \prod_{j=1}^{\delta} P\left(\alpha_{j}, y\right)
$$

is a nonzero integral polynomial with

$$
\begin{aligned}
& h t(Q) \leq e^{3 T \delta d_{p}} h_{p}^{\delta}\left(1+d_{p}\right)^{2 \delta}, \\
& \operatorname{deg} Q \leq \delta \cdot d_{p} .
\end{aligned}
$$

Our immediate goal is to estimate $|Q(\wp(\beta u))|$, which requires that we estimate $|P(\alpha, \wp(\beta u))|$ for each $j=1, \ldots, \delta$.

For $j=1$ we have

$$
\begin{aligned}
\left|P\left(\alpha_{1}, \wp(\beta u)\right)\right| \leq & \left|P\left(\alpha_{1}, \wp(\beta u)\right)-P(\wp(u), \wp(\beta u))\right|+|P(\wp(u), \wp(\beta u))| \\
\leq & h_{p}\left(1+d_{p}\right)^{3}(1+|\wp(u)|)^{d_{p}} \max \{1,|\wp(\beta u)|\}^{d_{p}}\left|a_{T}-\wp(u)\right| \\
& +e^{-t^{\varepsilon} M^{\varepsilon}} \\
\leq & c_{2}^{d_{p}} h_{p}\left(1+d_{p}\right)^{3} e^{-T^{\varepsilon}}
\end{aligned}
$$


by our choice of $T$. For the other choices of $j$ we have the general estimate

$$
\left|P\left(\alpha_{j}, \wp(\beta u)\right)\right| \leq h_{p}\left(1+d_{p}\right)^{2} \max \left\{1,\left|\alpha_{j}\right|\right\}^{d} \max \{1,|\wp(\beta u)|\}^{d_{p}} .
$$

If we then apply the estimate $\max \left\{1,\left|\alpha_{j}\right|\right\} \leq h t\left(a_{T}\right)+1 \leq e^{2 T}$, we deduce that for $j=2, \ldots, \delta$

$$
\left|P\left(\alpha_{j}, \wp(\beta u)\right)\right| \leq c_{3}^{d_{p}} h_{p}\left(1+d_{p}\right)^{2} e^{2 T d_{p}} .
$$

Combining (3) and (4) we deduce that

$$
\begin{aligned}
|Q(\wp(\beta u))| & \leq c_{4}^{\delta d_{p}} h_{p}^{\delta}\left(1+d_{p}\right)^{2 \delta+1} e^{3 T \delta d_{p}-T^{\ell}} \\
& \leq c_{4}^{\delta \cdot d_{p}} h_{p}^{\delta}\left(1+d_{p}\right)^{2 \delta+1} e^{-T^{\ell} / 2},
\end{aligned}
$$

provided $S_{1}$ is sufficiently large.

We next choose a root $\gamma$ of $Q(y)$ which is close to $\wp(\beta u)$. By Lemma 2 there exists such a $\gamma$, of multiplicity $m$, with

$$
\begin{aligned}
|\gamma-\wp(\beta u)|^{m} & \leq e^{7 d_{p}^{2} \delta^{2}} h t(Q)^{2 d_{p} \delta} \cdot|Q(\wp(\beta u))| \\
& \leq e^{c_{5} \delta^{2} d_{p}(T d p+\log h p)-T^{\varepsilon} / 2} .
\end{aligned}
$$

Since $1 \leq m \leq d_{p} \delta$, we deduce that

$$
\begin{aligned}
|\gamma-\wp(\beta u)| & \leq e^{c_{5}\left(T \delta d_{p}+\delta \log h_{p}\right)-T^{e} d_{p}^{-1} \delta^{-1} / 2} \\
& \leq e^{-T^{e} d_{p}^{-1} \delta^{-1} / 3}
\end{aligned}
$$

by our choice of $T$, provided $S_{1}$ is sufficiently large.

We recall that $\wp^{\prime}(\beta u) \neq 0$ and then apply Lemma 1 to choose $w_{T} \in \mathbf{C}$ such that $\wp\left(w_{T}\right)=\gamma$ and

$$
\left|w_{T}-\beta u\right|<k_{2} e^{-T^{\varepsilon} d_{p}^{-1} \delta^{-1} / 3} .
$$

Moreover, $\wp(u)$ is transcendental by a simple Liouville estimate; therefore $\wp^{\prime}(u) \neq 0$ and by Lemma 1 there exists $v_{T} \in \mathbf{C}$ such that $\wp\left(v_{T}\right)=a_{T}$ and

$$
\left|v_{T}-u\right|<k_{2} e^{-T^{\varepsilon}}
$$

Therefore,

$$
\left|w_{T}-\beta v_{T}\right| \leq c_{6} e^{-T^{\varepsilon} d_{p}^{-1} \delta^{-1} / 3} .
$$

Note that each of $\wp\left(w_{T}\right)$ and $\wp\left(v_{T}\right)$ is algebraic with

$$
\operatorname{deg} \wp\left(w_{T}\right) \leq \operatorname{deg} Q \leq \delta \cdot d_{p}, \quad \operatorname{deg} \wp\left(v_{T}\right)=\operatorname{deg} a_{T}=\delta
$$

and

$$
h t \wp\left(w_{T}\right) \leq 4^{\delta d p} h t Q, \quad h t \wp\left(v_{T}\right) \leq e^{T} .
$$

If $w_{T}-\beta v_{T}=0$, then $\wp\left(\beta v_{T}\right) \in \overline{\mathbf{Q}}$, which along with all of $g_{2}, g_{3}$, and $\wp\left(v_{T}\right)=a_{T}$ being algebraic contradicts the Schneider-Lang theorem, Theorem 1, p. 21, [4]. Therefore $w_{T}-\beta v_{T} \neq 0$ and we apply Lemma 3 with the 
following choices:

$$
\begin{gathered}
L=\mathbf{Q}\left(g_{2}, g_{3}, \beta, \wp\left(v_{T}\right), \wp\left(w_{T}\right)\right), \quad D=[L: \mathbf{Q}], \\
\log V_{1}=\log h t \gamma, \quad \log V_{2}=T, \quad B=D \max \{T, \log h t \gamma\}, \\
E=\min \left\{e(D \log h t \gamma)^{1 / 2} /\left|w_{T}\right|, \quad e(D T)^{1 / 2} /\left|v_{T}\right|\right\}
\end{gathered}
$$

to find that

$$
\left|w_{T}-\beta v_{T}\right|>\exp \left(-C_{3} D^{6}(\log B)^{3}(\log h t Q) T(\log D)(\log E)^{-4}\right) .
$$

Recalling our estimate for $\operatorname{deg} Q$ and $h t Q,(2)$ above and $D \leq \delta^{2} d_{p}$, we obtain

$$
\left|w_{T}-\beta v_{T}\right|>\exp \left(-c_{7} \delta^{13} d_{p}^{6} T\left(T d_{p}+\log h_{p}\right)(\log T)^{3}\right) \text {. }
$$

This last inequality contradicts (6), thanks to our choice of $T \geq M$, provided we take $S_{1}$ to be sufficiently large. Thus Theorem 2 is established.

As we mentioned above the proof of Theorem 4 follows the same outline as the proof of Theorem 2, hence we only indicate where the proofs differ.

Proof of Theorem 4. Suppose that $P(X, Y) \in \mathbf{Z}[X, Y]$ is a nonzero polynomial of total degree at most $d_{p}$ and height at most $h_{p}$ with

$$
|P(u, \wp(\beta u))| \leq \exp \left(-t_{1}^{\varepsilon} M_{1}^{\varepsilon}\right),
$$

here with $t_{1}$ and $M_{1}$ as in the statement of Theorem 4. As in the proof of Theorem 2 above, take $S_{2}$ sufficiently large so that there exists $a_{T}$ approximating $u$ with

$$
M_{1} \leq T \leq t_{1} M_{1} .
$$

Let $a_{T}=\alpha_{1}, \ldots, \alpha_{\delta}$ denote the conjugates of $a_{T}$.

Define $Q(y)$ as in (1) in the proof of Theorem 2, and note that $h t(Q)$ and $\operatorname{deg} Q$ satisfy the same estimates as before. To estimate $|Q(\wp(\beta u))|$ we now have

and for $j=2, \ldots, \delta$,

$$
\left|P\left(\alpha_{1}, \wp(\beta u)\right)\right| \leq c_{8}^{d_{p}} h_{p}\left(1+d_{p}\right)^{3} e^{-T^{\varepsilon}}
$$

$$
\left|P\left(\alpha_{j}, \wp(\beta u)\right)\right| \leq c_{9}^{d_{p}} h_{p}\left(1+d_{p}\right)^{2} e^{2 T d_{p}} .
$$

Combining these estimates we obtain

$$
|Q(\wp(\beta u))| \leq c_{10}^{\delta \cdot d_{p}} h_{p}^{\delta}\left(1+d_{p}\right)^{2 \delta+1} e^{-T^{\varepsilon} / 2},
$$

provided $S_{2}$ is sufficiently large.

We apply Lemma 2 to choose a root $\gamma$ of $Q(y)$ of multiplicity $m^{\prime}$ such that (5) holds with $m^{\prime}$ replacing $m$. Therefore, our choice of $T$ yields

$$
|\gamma-\wp(\beta u)|<e^{-T^{\varepsilon} d_{p}^{-1} \delta^{-1} / 3}
$$

provided $S_{2}$ is sufficiently large. We remark that $\wp^{\prime}(\beta u) \neq 0$, for otherwise $\wp(\beta u) \in \overline{\mathbf{Q}}$ and we apply Lemma 3 to $\beta_{0}=\beta a_{T}, u_{1}=\beta u$ to obtain

$$
\left|\beta a_{T}-\beta u\right|>\exp \left(-c_{12} \delta^{4}\right) \text {. }
$$


However this contradicts the upper bound

$$
\left|\beta a_{T}-\beta u\right|<|\beta| e^{-T^{\ell}},
$$

provided $S_{2}$ is taken sufficiently large, so that $T$ is sufficiently large. Using Lemma 1 we may then choose $w_{T} \in \mathbf{C}$ such that $\wp\left(w_{T}\right)=\gamma$ and

$$
\left|w_{T}-\beta u\right|<k_{2} e^{-T^{e} d_{p}^{-1} \delta^{-1} / 3} \text {. }
$$

Then

$$
\left|w_{T}-\beta a_{T}\right|<c_{11} e^{-T^{\varepsilon} d_{p}^{-1} \delta^{-1} / 3} .
$$

Note that $\wp\left(w_{T}\right)$ is algebraic with

$$
\operatorname{det} \wp\left(w_{T}\right) \leq \operatorname{deg} Q, \quad h t \wp\left(w_{T}\right) \leq 4^{\delta d p} h t Q
$$

and $\beta a_{T}$ is algebraic with

$$
\operatorname{deg} \beta a_{T} \leq(\operatorname{deg} \beta) d_{0}, \quad h t\left(\beta a_{T}\right) \leq e^{T \operatorname{deg} \beta} h t(\beta)^{d_{0}} .
$$

Moreover, if $w_{T}=\beta a_{T}$, then $w_{T}$ and $\wp\left(w_{T}\right)$ are simultaneously algebraic, which together with $g_{2}$ and $g_{3}$ being algebraic contradicts the Schneider-Lang theorem.

Therefore $w_{T}-\beta a_{T} \neq 0$ and we apply Lemma 3 with

$$
\begin{gathered}
L=\mathbf{Q}\left(g_{2}, g_{3}, \beta a_{T}, \wp\left(w_{T}\right)\right), \quad D=[L: \mathbf{Q}], \\
\log V_{1}=\log h t \gamma, \quad B=\max \left\{D \log V_{1}, e^{T}\right\}, \\
E=\min \left\{B^{D}, e\left(D \log V_{1}\right)^{1 / 2} /\left|w_{T}\right|\right\}
\end{gathered}
$$

to obtain

$$
\left|w_{T}-\beta a_{T}\right| \geq \exp \left(-c_{13} \delta^{9} d_{p}^{4} T^{2}\left(T d_{p}+\log h_{p}\right) .\right.
$$

If $S_{2}$ is taken to be sufficiently large, this last inequality contradicts (7). This established Theorem 4.

\section{REFERENCES}

1. W. D. Brownawell, On the Gelfond-Feldman measure of algebraic independence, Compositio Math. 38 (3) (1979), 355-368.

2. W. D. Brownawell and M. Waldschmidt, The algebraic independence of certain numbers to algebraic powers, Acta Arith. 32 (1977), 63-71.

3. N. I. Feldman, Arithmetic properties of the solutions of a transcendental equation, Vestnik Moskov. Univ. Ser. I, 1 (1964), 13-20.

4. S. Lang, Introduction to transcendental numbers, Addison-Wesley, Reading, MA, 1966.

5. M. Laurent, Indépendance Algébrique de nombres de Liouville à des puissances algébriques, These $3^{e}$ Cycle, L'Universite Pierre et Marie Curie, October 1977.

6. P. Philippon and M. Waldschmidt, Formes lineaires de logarithmes elliptiques et measures de transcendance, in Number Theory Proceedings of International Number Theory Conference, Quebec, de Gruyter, Berlin, 1989. 
7. R. Tubbs, A diophantine problem on elliptic curves, Trans. Amer. Math. Soc. 309 (1988), 325-338.

8. __ A transcendence measure for some special values of elliptic functions, Proc. Amer. Math. Soc. (2) 88 (1983), 189-196.

9. M. Waldschmidt, Nombres transcendants, Lecture Notes in Math., vol. 402, Springer-Verlag, New York, 1974.

Department of Mathematics, University of Colorado, Boulder, Colorado 80309 